\title{
The Usefulness of the Combination of D-Dimer and Soluble Fibrin Monomer Complex for Diagnosis of Venous Thromboembolism in Psychiatric Practice: A Prospective Study
}

\author{
Masahiro Takeshima (DD ${ }^{\prime}$ \\ Hiroyasu Ishikawa' \\ Masaya Ogasawara' \\ Munehiro Komatsu² \\ Dai Fujiwara' \\ Yu Itoh (iD) ${ }^{2}$ \\ Yuki Wada ${ }^{3}$ \\ Yuki Omori (D) ${ }^{4}$ \\ Hidenobu Ohta' \\ Kazuo Mishima (D) \\ 'Department of Neuropsychiatry, Akita \\ University Graduate School of Medicine, \\ Akita, Japan; ${ }^{2}$ Department of \\ Neuropsychiatry, Akita City Hospital, \\ Akita, Japan; ${ }^{3}$ Department of Radiology, \\ Akita University Graduate School of \\ Medicine, Akita, Japan; ${ }^{4}$ Department of \\ Neuropsychiatry, Tokyo Metropolitan \\ Geriatric Hospital, Tokyo, Japan
}

Purpose: D-dimer has the advantage of excluding venous thromboembolism (VTE) due to its high sensitivity but is disadvantageous for diagnosing VTE due to its low specificity. A method to increase the usefulness of D-dimer in the diagnosis of VTE is warranted. This study aimed to investigate the usefulness of the combination of D-dimer and soluble fibrin monomer complex (SFMC), which has been suggested as a new candidate marker for VTE, in VTE diagnosis.

Patients and Methods: This prospective study in 109 subjects was performed at a psychiatric department between August 1, 2017 and December 31, 2019. Subjects' levels of D-dimer and SFMC were measured simultaneously. Plasma levels of D-dimer and SFMC were measured using NANOPIA $\left.{ }^{(}\right)$D-dimer and NANOPIA ${ }^{\circledR}$ SF. Subjects with positive D-dimer $(\geq 1.0 \mu \mathrm{g} / \mathrm{mL})$ results underwent contrast computed tomography for confirmation of VTE within 12 hours of D-dimer measurement. A receiver operating characteristic curve analysis was performed to examine the usefulness of SFMC for the diagnosis of VTE.

Results: Only 109 of the 783 subjects without symptoms suggestive of VTE participated in the study. Out of 41 subjects with positive D-dimer results, 17 subjects were diagnosed with VTE. A receiver operating characteristic curve analysis was performed to determine cutoff values. The area under the curves was 0.848 for SFMC ( $p<0.001,95 \%$ CI 0.722 to 0.974$)$, and the optimal cutoff value was $10.0 \mu \mathrm{g} / \mathrm{mL}$ (sensitivity $58.8 \%$, specificity $100 \%$, positive predictive value $100 \%$, negative predictive value $77.4 \%$ ).

Conclusion: SFMC was useful for diagnosing VTE in the psychiatric patients with positive D-dimer results.

Keywords: computed tomography, D-dimer, psychiatric patients, soluble fibrin monomer complex, venous thromboembolism

\section{Introduction}

Venous thromboembolism (VTE) is a major cause of death among patients with psychiatric disorders. ${ }^{1}$ Schizophrenia, bipolar disorder, depression, antipsychotics, and antidepressants increase the risk of VTE. ${ }^{2-8}$ A recent population-based cohort study reported that the relative risk of deep vein thrombosis (DVT) was 3-fold higher, and that of pulmonary embolism (PE) was 2.6-fold higher, in patients with concurrent depressive, bipolar, and schizophrenic disorders than that in the general population. ${ }^{5}$ Previous cross sectional studies reported relatively high prevalence of
Correspondence: Masahiro Takeshima Department of Neuropsychiatry, Akita University Graduate School of

Medicine, I-I-I, Hondo, Akita City

Akita, 0I0-8543, Japan

Tel $+8|-| 8-884-6122$

Fax +8I-18-884-6445

Emailm.takeshima@med.akita-u.ac.jp 
VTE in patients hospitalized with psychiatric disorders, as $8.5 \%$ of patients with depression had VTE, ${ }^{9}$ and $11.6 \%$ of physically restrained patients ${ }^{10}$ and $25.3 \%$ of patients with catatonia had DVT. ${ }^{11}$ Further, previous studies reported that $62.5-76.9 \%$ of asymptomatic VTE cases were PE. ${ }^{9,12}$ Therefore, identifying psychiatric patients with a high VTE risk and conducting early VTE screening in such high-risk groups is crucial.

VTE is commonly screened using D-dimer testing. D-dimer has the advantage of a high negative predictive value of $97-100 \%$ and a high sensitivity of $93-100 \%$ when used in high-sensitivity assays for the diagnosis of VTE. ${ }^{13-17}$ However, D-dimer has a low positive predictive value and low specificity, ${ }^{13-17}$ and has therefore mainly been used for excluding VTE. ${ }^{16,18}$ If the screening is positive, VTE is definitively diagnosed by imaging, which is not always easy because psychiatric hospitals generally do not have sufficient laboratory equipment or skilled staff to diagnose VTE, and patients with severe mental disorders sometimes cannot be undergo imaging tests for VTE due to inability to maintain rest. Therefore, developing an easy and useful method for VTE diagnosis in the psychiatric field is warranted.

Soluble fibrin monomer complex (SFMC) is a serological marker for the activation of the coagulation system, and a quantitative SFMC testing method of plasma has been established. SFMC, like D-dimer, has been suggested as a marker for VTE. However, the results of previous studies examining the usefulness of SFMC for VTE diagnosis are inconsistent. In one study, setting the cutoff value of NANOPIA ${ }^{\circledR}$ SF to $5.9 \mu \mathrm{g} / \mathrm{mL}$ yielded a sensitivity of $98.5 \%$ and specificity of $80.1 \%$. The authors concluded that SFMC is useful for VTE diagnosis and exclusion. ${ }^{19}$ In another study, however, setting the cutoff value of NANOPIA ${ }^{\circledR}$ SF to $7.0 \mu \mathrm{g} /$ $\mathrm{mL}$ yielded a sensitivity of only $38.9 \%$ and a specificity of only $64.3 \%$, indicating that SFMC is not useful for VTE diagnosis or exclusion. ${ }^{20}$ In orthopedic surgery, an optimal cutoff level of $13.9 \mu \mathrm{g} / \mathrm{mL}$ yielded a sensitivity of $67.9 \%$ and a specificity of $78.2 \%{ }^{21}$ Although previous studies have not directly compared SFMC and D-dimer for VTE, SFMC seems to have a higher specificity than D-dimer. ${ }^{19-21}$

D-dimer has the disadvantage of low specificity; however, we hypothesized that the combination of D-dimer and SFMC would increase its usefulness for diagnosing VTE. Therefore, we conducted a prospective study to investigate this in psychiatric practice, using contrast computerized tomography (CT) for confirmation of VTE.

\section{Patients and Methods}

We performed this prospective study from August 2017 to December 2019 at the psychiatric department of our institution. Participants were recruited from consecutive inpatients with mental disorders during the study period. The inclusion criteria were patients with psychiatric disorders who were admitted at the psychiatric department of our institution for treatment of psychiatric disorder during the study periods. The exclusion criteria were: (1) iodine or iodine contrast agent sensitivity; (2) severe thyroid disease; (3) bronchial asthma; (4) severe renal dysfunction; (5) severe liver dysfunction; (6) severe heart failure; (7) macroglobulinemia; (8) multiple myeloma; (9) tetany; (10) pheochromocytoma; (11) age $<20$ years; and (12) pregnancy or nursing.

After participants agreed to participation in this study, their DVT risk was assessed using Well's score. ${ }^{22}$ Plasma levels of D-dimer and SFMC were measured using NANOPIA $^{\circledR}$ D-dimer and NANOPIA ${ }^{\circledR}$ SF (SEKISUI $^{\circ}$ MEDICAL CO., LTD., Tokyo, Japan). NANOPIA ${ }^{\circledR}$ D-dimer uses a monoclonal antibody specific for fragment DD, while NANOPIA ${ }^{\circledR}$ SF uses a monoclonal antibody J2-23, which recognizes an epitope in the C-terminal region of the fibrin $\mathrm{A} \alpha$ chain $(\mathrm{A} \alpha 502-521){ }^{23}$ The sensitivity of NANOPIA ${ }^{\circledR}$ D-dimer in diagnosing VTE has been reported as $99.6 \%$ with a cutoff value of $1.0 \mu \mathrm{g} /$ $\mathrm{mL}{ }^{24}$ The measuring equipment consisted of the automated coagulation analyzer COAPRESTA 2000 (Sekisui Medical Co., Ltd., Tokyo, Japan) until October 2019 and the COAPRESTA 3000 (Sekisui Medical Co., Ltd., Tokyo, Japan) from November 2019.

Subjects with positive D-dimer results underwent contrast CT within 12 hours of D-dimer measurements for the diagnosis of VTE. CT scans for detecting VTE (PE or DVT) were performed using multidetector row helical CT scanners (August 2017-January 2018: Discovery CT750HD or Discovery CT750HD-A; February 2018December 2019: Revolution CT; GE Healthcare Japan, Tokyo, Japan) with intravenously injected low-osmolar iodinated contrast mediums (the mainly used contrast mediums were Omnipaque 300; DAIICHI SANKYO COMPANY, Limited, Tokyo, Japan and Iomeron 350; Eisai Corporation, Limited, Tokyo, Japan). Slice thickness was $1.25 \mathrm{~mm}$. The total amount of contrast medium was determined according to body weight. Between 
August 2017 and January 2018, the total amount was calculated according to the formula "body weight $(\mathrm{kg}) \times$ $2+30$ (mL)," up to $150 \mathrm{~mL}$, for Omnipaque and according to the formula "body weight $(\mathrm{kg}) \times 2.5(\mathrm{~mL})$," up to $135 \mathrm{~mL}$, for Iomeron. Between February 2018 and December 2019, the total amount was calculated according to the formula "body weight $(\mathrm{kg}) \times 600 /$ iodine concentration of contrast medium $(\mathrm{mgI} / \mathrm{mL})$ "; for example, "body weight $(\mathrm{kg}) \times 600 / 350(\mathrm{~mL})$ " for Iomeron 350 and "body weight $(\mathrm{kg}) \times 600 / 300(\mathrm{~mL})$ " for Omnipaque 300. The contrast medium was intravenously injected at a rate of $3.5-4.0 \mathrm{~mL} / \mathrm{s}$ using a power injector, and scanning was performed at 20-30 seconds (early phase) for detecting pulmonary arterial embolization (CT angiography) and at 210-240 seconds (delayed phase) for detecting DVT (CT venography). These protocols were sometimes modified according to the patients' renal function, allergies to specific contrast media, and diameter of intravenous access. Image readings of CT scans were performed by radiological diagnostic specialists of the Japan Radiological Society or by doctors specializing in diagnosis who had exclusively worked at radiology departments for more than 10 years.

The following clinical characteristics of the participants were collected from their medical charts: age, sex, body mass index (BMI; $\mathrm{kg} / \mathrm{m}^{2}$ ), diagnosis of psychiatric disorders (International Classification of Diseases 10th Revision: ICD-10), restraint, catatonia according to the Diagnostic and Statistical Manual of Mental DisordersFifth Edition, daily dose of antipsychotic converted to chlorpromazine, daily dose of antidepressant converted to imipramine, ${ }^{25}$ history of VTE, anticoagulant use, D-dimer value $(\mu \mathrm{g} / \mathrm{mL})$, SFMC value, surgery within 4 weeks, trauma within 4 weeks, active cancer, infection, disseminated intravascular coagulation (DIC), hypertension, hyperlipidemia, diabetes mellitus, and fever $\left({ }^{\circ} \mathrm{C}\right)$.

\section{Calculation}

All statistical analyses were performed with IBM SPSS Statistics version 25.0 (IBM Corp., Chicago, IL, USA). Fisher's exact test was performed for nominal variables, and the Mann-Whitney $U$-test was used for continuous variables to examine the differences between the VTE and non-VTE subject groups. Using SFMC values and presence or absence of VTE in patients with positive D-dimer results, the usefulness of SFMC for the diagnosis of VTE was examined using receiver operating characteristic (ROC) curve analysis. Optimal cutoff value of SFMC was determined by the ROC analysis. The non-parametric (trapezoidal rule) area under the ROC curve was calculated to indicate overall accuracy. The McNemar chisquare test was used to estimate the statistical significance of differences in specificity when utilizing different threshold SFMC values.

\section{Power Analysis}

Power analysis was performed with $\mathrm{R}$ version 4.0.2. From previous studies, $(9,12)$ we estimated that one-third of psychiatric inpatients with a D-dimer value of $1 \mu \mathrm{g} / \mathrm{mL}$ or higher had VTE. Based on: (1) a one-sided significance level of 0.05 ; (2) power $=0.90$; (3) Area Under the Curve $(\mathrm{AUC})=0.8$; and (4) 1:3 ratio of the VTE-positive to the VTE-negative group, the minimum sample size to detect statistically significant differences was 38 subjects (10 subjects for the VTE-positive group and 28 subjects for the VTE-negative group, respectively). We therefore set a target sample size of 40 psychiatric inpatients with a D-dimer value of $1 \mu \mathrm{g} / \mathrm{mL}$ or higher.

\section{Ethics}

This study was approved by the Ethics Committee Akita University Graduate School of Medicine and Faculty of Medicine (No. 1821) and conducted in accordance with the principles of the Declaration of Helsinki. Written informed consent was obtained from each participant after a full explanation of the study protocol prior to study commencement.

\section{Results}

During the research period, 783 subjects were admitted to our hospital for the treatment of psychiatric disorders. Of these, 598 patients were excluded because of their refusal to participate, and 76 were excluded based on the exclusion criteria (2: iodine or iodine contrast agent sensitivity; 10: severe renal dysfunction; and 64: age $<20$ years). Finally, 109 subjects without VTE symptoms participated in the study. Forty-seven subjects showed positive test results (D-dimer $\geq 1.0 \mu \mathrm{g} / \mathrm{mL}$ ). SFMC was not measured in four subjects. Two patients were withdrawn from the study after the VTE screening: in one patient, severe renal dysfunction was revealed by a blood test conducted as part of the VTE screening and the other patient refused to undergo CT. Finally, 41 subjects underwent contrast CT. Participant selection is shown in Figure 1.

Of these 41 patients, 17 had VTE (DVT only: three, PE only: eight, both DVT and PE: six). Of all patients, DVT 


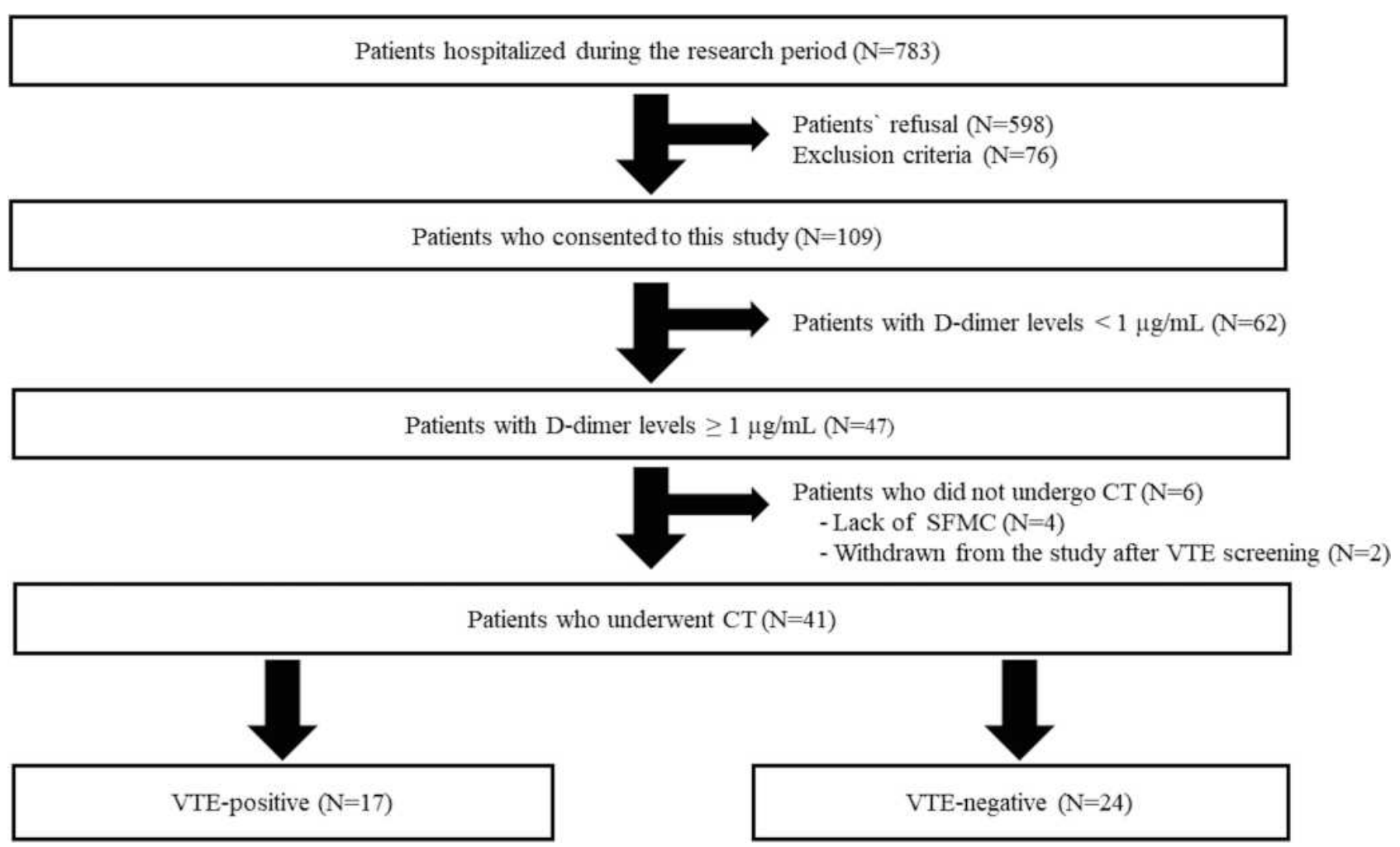

Figure I Selection of participants. During the research period, 783 subjects were admitted to our hospital for the treatment of psychiatric disorders. Of these, I09 subjects without venous thromboembolism (VTE) symptoms participated in the study, and 47 subjects showed positive test results (D-Dimer $\geq I$ ). SFMC was not measured in four subjects. Two patients were withdrawn from the study after the VTE screening. Finally, 4I subjects underwent contrast computerized tomography.

Abbreviations: SFMC, soluble fibrin monomer complex; VTE, venous thromboembolism.

was observed in $8.4 \%(8 / 95)$ of low-risk, $0 \%(0 / 12)$ of medium-risk, and $50 \%(1 / 2)$ of high-risk patients, categorized according to their Well's score.

The subjects' clinical characteristics are shown in Table 1. Compared to the VTE-negative group, the VTEpositive group had higher levels of D-dimer and SFMC $(p<0.001$ and $p<0.001)$ (see Figure 2). There were no differences between both groups in age, sex, BMI, operation within 4 weeks, trauma within 4 weeks, malignancy, infection, DIC, history of VTE, or use of anticoagulants.

The ROC analysis of SFMC is shown in Figure 3. The SFMC ROC curve had an AUC of $0.848(p<0.001,95 \%$ confidence interval [CI] 0.722 to 0.974$)$. The Youden index, used to automatically calculate the optimal cutoff value (sensitivity + specificity -1 ), was $10.0 \mu \mathrm{g} / \mathrm{mL}$ for SFMC (sensitivity $58.8 \%$, specificity $100 \%$, positive predictive value $100 \%$, negative predictive value $77.4 \%$ ).

\section{Discussion}

This is the first study to explore the usefulness of the combination of D-dimer and SFMC for the diagnosis of VTE. The results of this study provide cutoff values of SFMC that are sufficient for diagnosing VTE clinically, with a sensitivity of $58.8 \%$ and specificity of $100 \%$ among patients with psychiatric disorders and positive D-dimer results. Although previous studies have shown inconsistent results on the usefulness of SFMC, ${ }^{19-21}$ the ROC analysis conducted in the current study shows that SFMC is a useful marker for the diagnosis of VTE. It is unclear why the specificity of the cutoff value for SFMC in this study was high. Possible reasons that affected the results were that (1) VTE screening by D-dimer was performed simultaneously with SFMC measurement, and that (2) the subjects were psychiatric patients with few physical factors that elevate SFMC other than VTE compared to non-psychiatric patients. Since few studies have examined the usefulness of SFMC, further studies are warranted to validate these findings.

In a previous study using Well's score, 5\% (95\% CI 4 to $8 \%$ ) of low-risk, $17 \%$ (95\% CI 13 to $23 \%$ ) of middlerisk, and 53\% (95\% CI 44 to $61 \%$ ) of high-risk patients had DVT. ${ }^{22}$ In this study, these corresponding values were $8.4 \%, 0 \%$, and $50 \%$. Here, the prevalence of DVT was almost within range in the low-risk and high-risk groups, but it was lower in the middle-risk group than in the 
Table I Clinical and Demographic Characteristics

\begin{tabular}{|c|c|c|c|}
\hline & VTE-Positive $(n=\mid 7)$ & VTE-Negative $(n=24)$ & $p$-value \\
\hline D-dimer $(\mu \mathrm{g} / \mathrm{mL})^{\dagger}$ & $4.43(2.56-8.36)$ & I.7I (I.4I-2.6I) & $p=0.000 *$ \\
\hline SFMC $(\mu \mathrm{g} / \mathrm{mL})^{\dagger}$ & $10.6(5.7-34.5)$ & $3.5(2.3-6.2)$ & $p=0.000 *$ \\
\hline Age, $\mathrm{yr}^{\dagger}$ & $67(58-78)$ & $68.5(56.25-76.5)$ & $p=0.863$ \\
\hline $\begin{array}{l}\text { Sex }{ }^{\ddagger} \\
\text { Male } \\
\text { Female }\end{array}$ & $\begin{array}{l}7 \\
10\end{array}$ & $\begin{array}{l}8 \\
16\end{array}$ & $p=0.745$ \\
\hline BMI $\left(\mathrm{kg} / \mathrm{m}^{2}\right)^{\dagger}$ & $21.2(18.8-24.3)$ & $20.0(19.2-23.65)$ & $p=0.853$ \\
\hline \multicolumn{4}{|c|}{ Psychiatric disorders (ICD-I0) } \\
\hline $\begin{array}{l}\text { F0 } \\
\text { FI } \\
\text { F2 } \\
\text { F3 }\end{array}$ & $\begin{array}{l}1 \\
0 \\
2 \\
14\end{array}$ & $\begin{array}{l}6 \\
1 \\
2 \\
15\end{array}$ & \\
\hline Restraint $^{\ddagger}$ & 4 & 7 & $p=0.736$ \\
\hline Catatonia $^{\ddagger}$ & 2 & 1 & $p=0.560$ \\
\hline Antidepressants ${ }^{\ddagger}$ & 10 & 12 & $p=0.752$ \\
\hline IMPE $^{\dagger}$ & $100(0-188)$ & $18.8(0-100)$ & $p=0.301$ \\
\hline $\mathrm{CPZE}^{\dagger}$ & $0(0-250)$ & $100(0-302)$ & $p=0.354$ \\
\hline Surgery $<4 \mathrm{w}^{\ddagger}$ & 0 & 0 & Not calculated \\
\hline Trauma $<4 \mathrm{w}^{\ddagger}$ & 1 & 1 & $p=1.000$ \\
\hline Active cancer ${ }^{\ddagger}$ & 3 & 2 & $p=0.633$ \\
\hline Infection ${ }^{\ddagger}$ & 0 & 2 & $p=0.502$ \\
\hline $\mathrm{DIC}^{\ddagger}$ & 0 & 0 & Not calculated \\
\hline History of VTE ${ }^{\ddagger}$ & 1 & 1 & $p=1.000$ \\
\hline Anticoagulants ${ }^{\ddagger}$ & 1 & 1 & $p=1.000$ \\
\hline $\mathrm{HT}^{\ddagger}$ & 7 & 10 & $p=1.000$ \\
\hline $\mathrm{HL}^{\ddagger}$ & 3 & 7 & $p=0.480$ \\
\hline $\mathrm{DM}^{\ddagger}$ & 2 & 4 & $p=1.000$ \\
\hline Fever $\left({ }^{\circ} \mathrm{C}\right)^{\dagger}$ & $36.7(36.3-36.8)$ & $36.7(36.4-36.9)$ & $p=0.894$ \\
\hline
\end{tabular}

Notes: Values are presented as medians (25-75\% percentile). Significant $p$-values are labeled with an asterisk. 'Mann-Whitney $U$-test; ${ }^{\dagger}$ Fisher's exact test.

Abbreviations: BMI, body mass index; CPZE, chlorpromazine equivalents; DM, diabetes mellitus; ICD-I0, International Classification of Diseases I0th Revision; IMPE, imipramine equivalents; HL, hyperlipidemia; HT, hypertension; SFMC, soluble fibrin monomer complex; VTE, venous thromboembolism.

respective patient groups in the previous study. The reason for these differences between studies is unclear, but the small sample size may have affected the results of this study. Studies with larger sample sizes are warranted to determine whether Well's score is also useful in the evaluation of patients with psychiatric disorders.

\section{Strengths and Weaknesses of the Study}

The strengths of this study are (1) its prospective design, and (2) the detailed examination for VTE (both DVT and PE) using contrast-enhanced CT. Nevertheless, this study also has some limitations: (1) Only 109 of the 783 (13.9\%) patients admitted to our department participated in the study. 

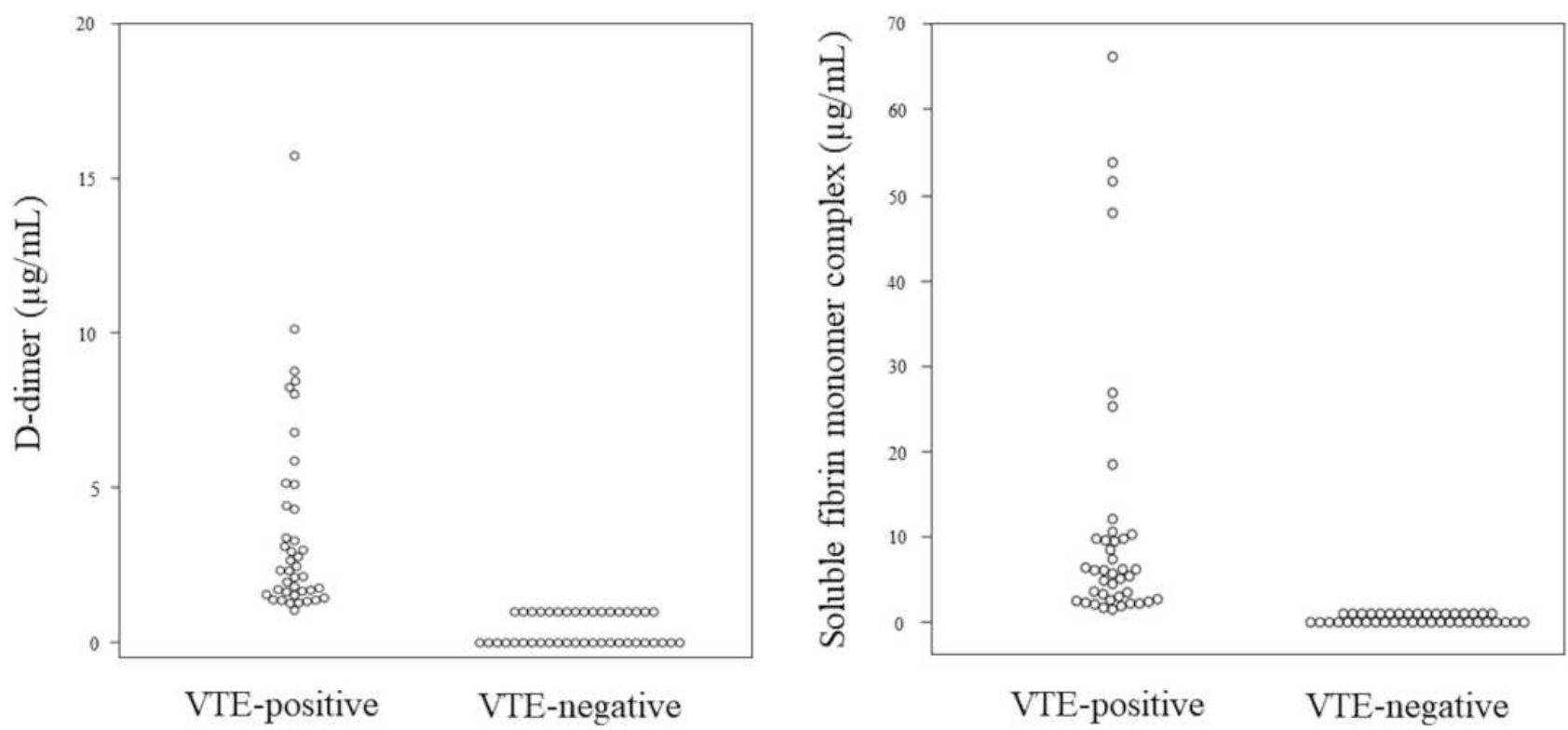

Figure 2 D-dimer and SFMC values in VTE-positive and VTE-negative patients. Bee swarm diagram showing the distribution of venous thromboembolism (VTE)-positive and VTE-negative patients for respective D-dimer and soluble fibrin monomer complex values.

Abbreviation: VTE, venous thromboembolism.

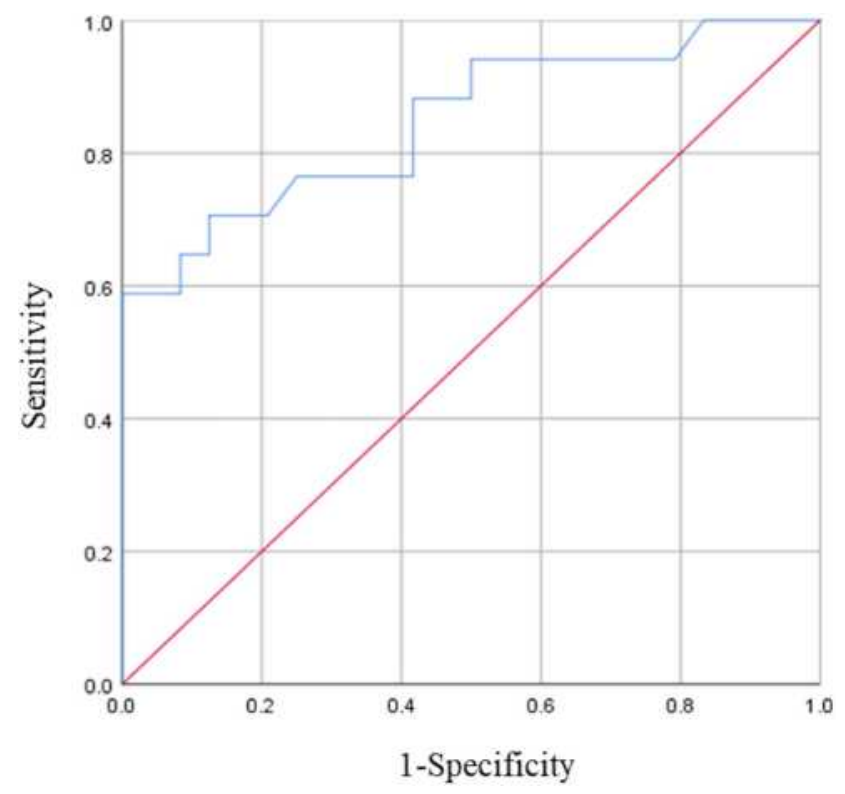

Figure 3 Receiver-operator characteristic curve (ROC) for predicting the possibility of venous thromboembolism based on soluble fibrin monomer complex (SFMC) values. The area under the curve was 0.848 for SFMC $(95 \% \mathrm{Cl} 0.722$ to 0.974 , $p<0.001$ ).

Selection bias may have affected the results of this study. (2) VTE may have been missed because patients with D-dimer levels $<1.0 \mu \mathrm{g} / \mathrm{mL}$ did not undergo contrast-enhanced CT, based on the criteria for the 1st screening; and (3) the ROC curve of D-dimer or SFMC alone could not be plotted because contrast-enhanced CT was not performed in patients with negative D-dimer results; (4) some participants might exhibit confounding factors that increase D-dimer or SFMC levels; (5) although a fixed 1.0 D-dimer level was used, the age-adjusted D-dimer level, which is recommended by several guidelines, ${ }^{26}$ may be more useful in combination with SFMC. These methodological issues should be resolved through future carefully designed studies.

\section{Conclusion}

SFMC was useful for diagnosing VTE in the psychiatric patients with positive D-dimer results.

\section{Abbreviations}

AUC, area under the curve; BMI, body mass index; CI, confidence interval; CT, computerized tomography; DIC, disseminated intravascular coagulation; DVT, deep vein thrombosis; PE, pulmonary embolism; ROC, receiver operating characteristic; SFMC, soluble fibrin monomer complex; VTE, venous thromboembolism.

\section{Data Sharing Statement}

The datasets used and analyzed in the current study are available from the corresponding author on reasonable request.

\section{Acknowledgments}

We would like to thank Editage (www.editage.jp) for English language editing. 


\section{Author Contributions}

All authors made substantial contributions to the conception and design of the study and to the acquisition of data or the analysis and interpretation of data; they all took part in drafting the article or revising it critically for important intellectual content; they gave their final approval of the version to be published; and all authors agree to be accountable for all aspects of the work.

\section{Funding}

This research did not receive any specific grant from funding agencies in the public, commercial, or not-forprofit sectors.

\section{Disclosure}

The authors report no conflicts of interest in this work.

\section{References}

1. Manu P, Kane JM, Correll CU. Sudden deaths in psychiatric patients. J Clin Psychiatry. 2011;72(7):936-941. doi:10.4088/JCP.10m06 244gry

2. Barbui C, Conti V, Cipriani A. Antipsychotic drug exposure and risk of venous thromboembolism: a systematic review and meta-analysis of observational studies. Drug Saf. 2014;37(2):79-90. doi:10.1007/ s40264-013-0127-6

3. Hsu WY, Lane HY, Lin CL, Kao CH. A population-based cohort study on deep vein thrombosis and pulmonary embolism among schizophrenia patients. Schizophr Res. 2015;162(1-3):248-252. doi:10.1016/j.schres.2015.01.012

4. Kunutsor SK, Seidu S, Khunti K. Depression, antidepressant use, and risk of venous thromboembolism: systematic review and meta-analysis of published observational evidence. Ann Med. 2018;50(6):529-537. doi:10.1080/07853890.2018.1500703

5. Lin CE, Chung $\mathrm{CH}$, Chen LF, Chien WC. Increased risk for venous thromboembolism among patients with concurrent depressive, bipolar, and schizophrenic disorders. Gen Hosp Psychiatry. 2019;61:34-40. doi:10.1016/j.genhosppsych.2019.10.003

6. Parkin L, Balkwill A, Sweetland S, et al. Antidepressants, depression, and venous thromboembolism risk: large prospective study of UK women. $J$ Am Heart Assoc. 2017;6(5):e005316. doi:10.1161/ JAHA.116.005316

7. Wu CS, Lin CC, Chang CM, et al. Antipsychotic treatment and the occurrence of venous thromboembolism: a 10-year nationwide registry study. J Clin Psychiatry. 2013;74(9):918-924. doi:10.4088/ JCP. $12 \mathrm{~m} 08117$

8. Zhang R, Dong L, Shao F, Tan X, Ying K. Antipsychotics and venous thromboembolism risk: a meta-analysis. Pharmacopsychiatry. 2011;44(5):183-188. doi:10.1055/s-0031-1280814

9. Takeshima M, Ishikawa H, Umeta Y, et al. Prevalence of asymptomatic venous thromboembolism in depressive inpatients. Neuropsychiatr Dis Treat. 2020;16:579-587. doi:10.2147/NDT.S243308

10. Ishida T, Katagiri T, Uchida $H$, et al. Incidence of deep vein thrombosis in restrained psychiatric patients. Psychosomatics. 2014;55 (1):69-75. doi:10.1016/j.psym.2013.04.001

11. Ishida T, Sakurai H, Watanabe K, Iwashita S, Mimura M, Uchida H. Incidence of deep vein thrombosis in catatonic patients: a chart review. Psychiatry Res. 2016;241:61-65. doi:10.1016/j. psychres.2016.04.105
12. Takeshima M, Ishikawa H, Shimizu K, Kanbayashi T, Shimizu T. Incidence of venous thromboembolism in psychiatric inpatients: a chart review. Neuropsychiatr Dis Treat. 2018;14:1363-1370. doi:10.2147/NDT.S162760

13. Di Nisio M, Squizzato A, Rutjes AW, Buller HR, Zwinderman AH, Bossuyt PM. Diagnostic accuracy of D-dimer test for exclusion of venous thromboembolism: a systematic review. J Thromb Haemost. 2007;5(2):296-304. doi:10.1111/j.1538-7836.2007.02328.x

14. Fronas SG, Wik HS, Dahm AEA, et al. Safety of D-dimer testing as a stand-alone test for the exclusion of deep vein thrombosis as compared with other strategies. J Thromb Haemost. 2018;16 (12):2471-2481. doi:10.1111/jth.14314

15. Ghanima W, Abdelnoor M, Mowinckel MC, Sandset PM. The performance of STA-Liatest D-dimer assay in out-patients with suspected pulmonary embolism. Br J Haematol. 2006;132(2):210-215. doi:10.1111/j.1365-2141.2005.05859.x

16. Vermeer HJ, Ypma P, Van Strijen MJL, et al. Exclusion of venous thromboembolism: evaluation of D-Dimer PLUS for the quantitative determination of D-dimer. Thromb Res. 2005;115(5):381-386. doi:10.1016/j.thromres.2004.09.005

17. Waser G, Kathriner S, Wuillemin WA. Performance of the automated and rapid STA Liatest D-dimer on the STA-R analyzer. Thromb Res. 2005;116(2):165-170. doi:10.1016/j.thromres.2004.12.003

18. Stein PD, Hull RD, Patel KC, et al. D-dimer for the exclusion of acute venous thrombosis and pulmonary embolism: a systematic review. Ann Intern Med. 2004;140(8):589-602. doi:10.7326/00034819-140-8-200404200-00005

19. Tsuji A, Wada H, Matsumoto T, et al. Elevated levels of soluble fibrin in patients with venous thromboembolism. Int $J$ Hematol. 2008;88 (4):448-453. doi:10.1007/s12185-008-0173-5

20. Yano S, Yoshida Y, Notsu Y, et al. Significance of D-dimer and soluble fibrin testing in screening of incident venous thromboembolism. Vasc Fail. 2019;3(1):26-30. doi:10.30548/ vascfail.3.1_26

21. Hasegawa $M$, Wada $H$, Wakabayashi $H$, et al. The relationships among hemostatic markers, the withdrawal of fondaparinux due to a reduction in hemoglobin and deep vein thrombosis in Japanese patients undergoing major orthopedic surgery. Clin Chim Acta. 2013;425:109-113. doi:10.1016/j.cca.2013.07.009

22. Wells PS, Owen C, Doucette S, Fergusson D, Tran H. Does this patient have deep vein thrombosis? JAMA. 2006;295(2):199-207. doi:10.1001/jama.295.2.199

23. Suzuki A, Ebinuma H, Matsuo M, Miyazaki O, Yago H. The monoclonal antibody that recognizes an epitope in the $\mathrm{C}$-terminal region of the fibrinogen $\alpha$-chain reacts with soluble fibrin and fibrin monomer generated by thrombin but not with those formed as plasmin degradation products. Thromb Res. 2007;121(3):377-385. doi:10.1016/j. thromres.2007.05.008

24. Yamaki T, Nozaki M, Sakurai H, et al. Combined use of pretest clinical probability score and latex agglutination D-dimer testing for excluding acute deep vein thrombosis. J Vasc Surg. 2009;50 (5):1099-1105. doi:10.1016/j.jvs.2009.06.059

25. Inada $T$, Inagaki A. Psychotropic dose equivalence in Japan. Psychiatry Clin Neurosci. 2015;69(8):440-447. doi:10.1111/ pcn. 12275

26. Konstantinides SV, Meyer G, Becattini C, et al. 2019 ESC Guidelines for the diagnosis and management of acute pulmonary embolism developed in collaboration with the European Respiratory Society (ERS): the Task Force for the diagnosis and management of acute pulmonary embolism of the European Society of Cardiology (ESC). Eur Respir J. 2019;54(3):1901647. doi:10.1183/13993003.016472019 


\section{Publish your work in this journal}

Vascular Health and Risk Management is an international, peerreviewed journal of therapeutics and risk management, focusing on concise rapid reporting of clinical studies on the processes involved in the maintenance of vascular health; the monitoring, prevention and treatment of vascular disease and its sequelae; and the involvement of metabolic disorders, particularly diabetes. This journal is indexed on PubMed Central and MedLine. The manuscript management system is completely online and includes a very quick and fair peerreview system, which is all easy to use. Visit http://www.dovepress. com/testimonials.php to read real quotes from published authors. 